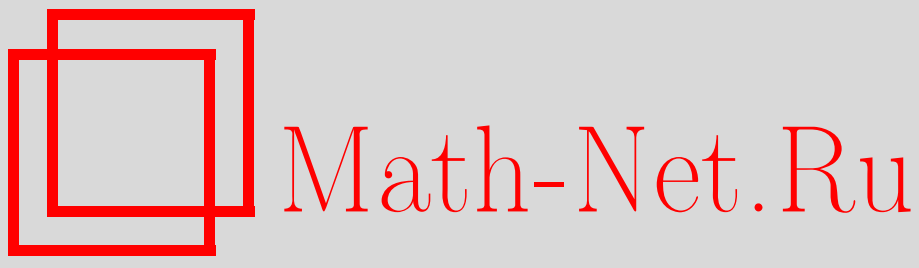

А. В. Пухликов, Бирационально жесткие гиперповерхности Фано, Изв. РАН. Сер. матем., 2002, том 66, выпуск 6, 159-186

DOI: https://doi.org/10.4213/im413

Использование Общероссийского математического портала Math-Net.Ru подразумевает, что вы прочитали и согласны с пользовательским соглашением

http://www . mathnet.ru/rus/agreement

Параметры загрузки:

IP : 35.174 .16 .151

26 апреля 2023 г., 05:03:02 
УДК 512.9

\title{
А.В. Пухликов
}

\section{Бирационально жесткие гиперповерхности Фано}

\begin{abstract}
Доказана бирациональная жесткость гладкой гиперповерхности Фано $V=$ $V_{M} \subset \mathbb{P}^{M}, M \geqslant 6$. В частности, она не может быть расслоена нетривиальным рациональным отображением на унилинейчатые многообразия, и любое ее бирациональное отображение на минимальное многообразие Фано той же размерности есть бирегулярный изоморфизм. Доказательство получено сочетанием метода максимальных особенностей и принципа связности Шокурова-Коллара.

Библиография: 29 наименований.
\end{abstract}

\section{Введение}

0.1. Основной результат. Зафиксируем целое число $M \geqslant 6$. Пусть $\mathbb{P}=\mathbb{P}^{M}$ комплексное проективное пространство, $V=V_{M} \subset \mathbb{P}$ - гладкая гиперповерхность степени $M$. Очевидно, $V \subset \mathbb{P}$ - многообразие Фано. По теореме Лефшеца $\operatorname{Pic} V=\mathbb{Z} K_{V}=\mathbb{Z} H$, где $K_{V}=-H, H$ - класс гиперплоского сечения на многообразии $V$. Основным результатом настояшей работы является следующая теорема.

ТЕоремА. Многообразие $V$ бирационально сверхжесткое.

СлЕДСТвИЕ. (i) Многообразие $V$ не может быть расслоено нетривиальнымм рациональным отображсением на унилинейчатые многообразия.

(ii) Любое бирачиональное отображсение $\chi: V \rightarrow-\rightarrow V^{\prime}$ на многообразие Фано $V^{\prime}$ c $\mathbb{Q}$-факториальными терминальными особенностями и группой Пикара $\operatorname{Pic} V^{\prime} \otimes \mathbb{Q}=\mathbb{Q} K_{V^{\prime}}$ есть (бирегулярный) изоморфизм. В частности, группы бирачиональных и бирегулярных автоморфизмов совпадают:

$$
\operatorname{Bir} V=\operatorname{Aut} V \text {. }
$$

(iii) Многообразие $V$ нерачионально.

Таким образом, основной результат работы дает многомерное обобщение знаменитой статьи [1], которая положила начало современной бирациональной геометрии. В.А. Исковских и Ю.И. Манин доказали бирациональную сверхжесткость трехмерных квартик (случай $M=4$ в наших обозначениях). Случай $M=5$ был рассмотрен в [14]. Сверхжесткость общих (в смысле топологии Зарисского) гиперповерхностей $V$ была доказана в [16]. Объединяя результаты работ [1], [14] и настоящей статьи, получаем бирациональную сверхжесткость всех гладких гипер-

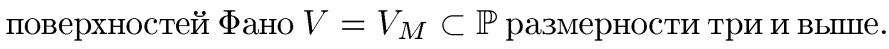

Работа выполнена при финансовой поддержке Российского фонда фундаментальных исследований (гранты № 02-15-99258, № 02-01-00441) и INTAS (грант INTAS-OPEN 2000-269).

(C) А.В. Пухликов, 2002 
0.2. Бирационально жесткие многообразия. В настоящей работе основным полем считается поле комплексных чисел $\mathbb{C}$. Напомним (см. [15]-[21]), что гладкое многообразие $\Phi$ ано $X$ размерности не менее 3 с числом Пикара $\operatorname{rk} \operatorname{Pic} X=1$ называется бирачионально сверхжестким, если для любого бирационального отображения

$$
\chi: X--\rightarrow X^{\prime}
$$

на многообразие $X^{\prime}$ той же размерности, гладкое в коразмерности 1, и любой линейной системы $\Sigma^{\prime}$ на $X^{\prime}$, свободной в коразмерности 1 (т. е. codim Bs $\Sigma^{\prime} \geqslant 2$ ), выполнено неравенство

$$
c(\Sigma, X) \leqslant c\left(\Sigma^{\prime}, X^{\prime}\right),
$$

где $\Sigma=\left(\chi^{-1}\right)_{*} \Sigma^{\prime}-$ собственный прообраз системы $\Sigma^{\prime}$ на $X$ относительно $\chi$ и $c(\Sigma, X)=c(D, X)$ обозначает порог канонического присоединения,

$$
c(D, X)=\sup \left\{b / a\left|b, a \in \mathbb{Z}_{+} \backslash\{0\},\right| a D+b K_{X} \mid \neq \varnothing\right\},
$$

$D \in \Sigma$. Аналогичным образом определяется $c\left(\Sigma^{\prime}, X^{\prime}\right)$.

Если для каждой тройки $\left(X^{\prime}, \chi, \Sigma^{\prime}\right)$ сушествует бирациональный автоморфизм $\chi^{*} \in \operatorname{Bir} X$ такой, что вместо неравенства (1) выполнено неравенство

$$
c\left(\Sigma^{*}, X\right) \leqslant c\left(\Sigma^{\prime}, X^{\prime}\right)
$$

где линейная система $\Sigma^{*}=\left(\chi \circ \chi^{*}\right)_{*}^{-1} \Sigma^{\prime}=\left(\chi^{*}\right)_{*}^{-1} \Sigma$ есть собственньй прообраз линейной системы $\Sigma^{\prime}$ относительно композиции

$$
\chi \circ \chi^{*}: X \stackrel{\chi^{*}}{\rightarrow} X \stackrel{\chi}{\rightarrow} \rightarrow X^{\prime},
$$

то $X$ называется бирационально жестким.

Хорошо известно, что бирационально жесткое многообразие $X$ не может быть расслоено нетривиальным рациональным отображением на унилинейчатые многообразия меньшей размерности (этот факт есть немедленное следствие оценки (2) с учетом предположения, что $\operatorname{Pic} X \cong \mathbb{Z}$; см. [16]) и что если $\chi: X--\rightarrow X^{\prime}$ - бирациональное отображение на многообразие $\Phi$ ано $X^{\prime}$ с $\mathbb{Q}$-факториальными терминальными особенностями и группой Пикара $\operatorname{Pic} X^{\prime} \otimes \mathbb{Q}=\mathbb{Q} K_{X^{\prime}}$, то $X^{\prime}$ (бирегулярно) изоморфно $X$ (см., например, [20]). В частности, бирационально жесткие многообразия нерациональны.

Бирациональная жесткость и сверхжесткость могут быть определены и для расслоений на многообразия Фано (см. [17], [19] и также [23]). Однако эта относительная версия понятия жесткости не обсуждается в настоящей работе.

Бирациональная (сверх)жесткость является одним из наиболее поразительных и загадочных феноменов многомерной алгебраической геометрии. Говоря неформально, жесткость означает, что алгебраические многообразия, не имеющие глобальных ненулевых дифференциальных форм (рационально связные многообразия), ведут себя так, как будто бы на них было много дифференциальных форм. В самом деле, из основного результата настоящей работы вытекает, что для любой гладкой гиперповерхности $V^{\prime} \subset \mathbb{P}$ любое бирациональное отображение

$$
\chi: V--\rightarrow V^{\prime}
$$


есть (проективный) изоморфизм. Если бы степень гиперповерхности $V$ была выше, чем $M+1$, то это утверждение было бы очевидным: достаточно сравнить очень обильную каноническую систему $\left|K_{V}\right|$ и систему $\left|K_{V^{\prime}}\right|$. Однако в нашем случае каноническая линейная система $\left|K_{V}\right|$ (вместе со всеми плюриканоническими линейными системами $\left.\left|m K_{V}\right|, m \geqslant 1\right)$ пуста, так что сравнивать нечего. И тем не менее, свойства гиперповерхности $V$ больше похожи на свойства многообразия общего типа, чем на свойства проективного пространства, близкого соседа гиперповерхности $V$ в классе многообразий Фано. Какие глубинные причины лежат в основе феномена бирациональной жесткости? Ответ на этот вопрос еще предстоит найти.

0.3. Логканонические особенности. Напомним некоторые определения теории логминимальных моделей. Пусть $X$ - неособое алгебраическое многообразие, $D=\sum_{i \in I_{D}} d_{i} D_{i}-\mathbb{Q}$-дивизор, где $0<d_{i}<1, D_{i} \subset X$ - попарно различные неприводимые гиперповерхности.

ОПРЕДЕЛЕНИЕ 1. Неприводимое подмногообразие $W \subset X$ называется логцентром пары $(X, D)$, если для некоторого разрешения особенностей $f: Y \rightarrow X$ пары $(X, D)$ со множеством $\left\{E_{i} \subset Y \mid i \in I_{f}\right\}$ исключительных дивизоров имеем представление

$$
K_{Y}+\widetilde{D}=f^{*}\left(K_{X}+D\right)+\sum_{i \in I_{f}} e_{i} E_{i}
$$

где для некоторого $i \in I_{f}$ выполнено

$$
f\left(E_{i}\right)=W \quad \text { и } \quad e_{i} \leqslant-1 .
$$

Множество логцентров не является инвариантом пары $(X, D)$. Однако теоретико-множественное объединение всех логцентров

$$
\mathrm{LC}(X, D)=\bigcup_{e_{i} \leqslant-1} f\left(E_{i}\right)
$$

зависит только от пары $(X, D)$. Для произвольной точки $x \in X$ обозначим символом $\mathrm{LC}(X, D, x)$ связную компоненту замкнутого алгебраического множества $\mathrm{LC}(X, D)$, содержащую $x$.

ОПРЕДЕЛЕНИЕ 2. Пара $(X, D)$ логтерминальна в точке $x$ в размерности $l \geqslant 1$, если

$$
\operatorname{dim} \mathrm{LC}(X, D, x) \leqslant l-1
$$

Пара $(X, D)$ логтерминальна в точке $x$, если $x \notin \mathrm{LC}(X, D)$, или, что то же camoe, $\mathrm{LC}(X, D, x)=\varnothing$. 
ОПРЕДЕЛЕНИЕ 3 . Пара $(X, D)$ логканонична в точке $x$ в размерности $l \geqslant 1$, если пара

$$
\left(X, \frac{1}{1+\varepsilon} D\right)
$$

логтерминальна в точке $x$ в размерности $l$ для всех достаточно малых $\varepsilon>0$.

Аналогичньм образом определяется логканоничность в точке $x$.

Теперь рассмотрим пару $\left(\mathbb{P}^{k}, D\right)$. Естественным образом определим степень $\mathbb{Q}$-дивизора $D$ как число

$$
\operatorname{deg} D=\sum_{i \in I_{D}} d_{i} \operatorname{deg} D_{i},
$$

так что $D \equiv(\operatorname{deg} D) H$ в $A^{1} \mathbb{P}^{k} \otimes \mathbb{Q}$.

ПРЕДЛОЖЕНИЕ 1. Если пара $\left(\mathbb{P}^{k}, D\right)$ логтерминальна (соответственно, логканонична) в точке $x \in \mathbb{P}^{k}$ в размерности $l \geqslant 1$, причем челое число $l$ удовлетворяет неравенству

$$
l+\operatorname{deg} D \leqslant k+1
$$

то она логтерминальна (соответственно, логканонична) в точке $x$.

Доказательство дано в $\S 2$.

0.4. Максимальные особенности. Предположим, что существуют бирациональное отображение

$$
\chi: V--\rightarrow V^{\prime}
$$

на многообразие $V^{\prime}$ той же размерности, гладкое в коразмерности 1 , и подвижная линейная система $\Sigma^{\prime}$ на $V^{\prime}$ такие, что для собственного прообраза $\Sigma$ системы $\Sigma^{\prime}$ выполнено неравенство

$$
c(\Sigma, V)>c\left(\Sigma^{\prime}, V^{\prime}\right) .
$$

Очевидно, $c(\Sigma, V)=n \geqslant 1$, где $\Sigma \subset|n H|$.

ПРЕДЛОЖЕНИЕ 2. В сделанных выше предположениях существует геометрическое дискретное нормирование $\nu$ (т. е. нормирование, дивизориальное на некоторой модели $V^{\sharp}$ многообразия $V$ ), удовлетворяющее неравенству Нётера-Фано

$$
\nu(\Sigma)>n \cdot \operatorname{discrepancy}(\nu) .
$$

ОПРЕДЕЛЕниЕ 4. Геометрическое дискретное нормирование, удовлетворяющее неравенству Нётера-Фано (4), называется максимальной особенностью линейной системы $\Sigma$.

В явном виде неравенство (4) означает, что существуют бирациональный морфизм $\varphi: V^{\sharp} \rightarrow V$ проективных многообразий и простой дивизор $E \subset V^{\sharp}$ такие, что $E \not \subset \operatorname{Sing} V^{\sharp}$ и

$$
\operatorname{ord}_{E}\left(\varphi^{*} \Sigma\right)>n a(E)
$$

где $a(E)$ обозначает дискрепантность дивизора $E$ относительно модели $V$. 
ДОКАЗАТЕЛЬСТВО ПРЕДЛОЖЕНИЯ 2 содержится во многих работах (см., например, [1], [2], [4], [10], [11], [15]-[19]). Оно вполне элементарно.

Легко видеть, что центр $B=\varphi(E)$ максимальной особенности $\nu$ на $V$ удовлетворяет неравенству

$$
\operatorname{mult}_{B} \Sigma>n \text {. }
$$

ЗАмЕчАнИЕ 1. Если упомянутый выше морфизм $\varphi$ является просто раздутием неприводимого подмногообразия $B \subset V$, то условие того, что исключительный дивизор $E \subset V^{\sharp}$ есть максимальная особенность, принимает очень простой вид:

$$
\operatorname{mult}_{B} \Sigma>n(\operatorname{codim} B-1) \text {. }
$$

Этот частный случай неравенства Нётера-Фано называется неравенством Фано, и подмногообразие $B \subset V$ в этом случае назьвается максимальным подмногообразием (точкой, кривой, поверхностью, ... ) линейной системы $\Sigma$. Общая теория обеспечивает сушествование максимальной особенности, но не ее наиболее простого частного случая - максимального подмногообразия. Действительно, имеются примеры (см. [7]-[9]), когда максимального подмногообразия нет, в то время как легко найти максимальную особенность, сделав несколько раздутий. Если максимальная особенность реализована несколькими раздутиями (т. е. более чем одним), она называется бесконечно близкой.

Иными словами, общая теория дает существование либо максимального подмногообразия, либо бесконечно близкой максимальной особенности. Легко понять, что именно бесконечно близкий случай порождает наибольшие трудности; чтобы с ними справиться, иногда требуются поистине огромные усилия. Во всяком случае, читателю следует иметь в виду эту альтернативу, чтобы избежать путанищы в терминологии, к которой могут привести препринты [7]-[9].

0.5. Схема доказательства. Предположим, что многообразие $V$ не является бирационально жестким. В силу сказанного в предыдущем пункте и определения сверхжесткости отсюда следует, что существует подвижная линейная система $\Sigma \subset$ $|n H|$ с максимальной особенностью $\nu$. Прежде всего, отметим следуюший факт.

ПРЕДЛОЖЕНИЕ 3. Для любой кривой $C \subset V$ выполнено неравенство

$$
\operatorname{mult}_{C} \Sigma \leqslant n
$$

ДоКАЗАТЕЛЬСТво см. в [15], [16].

Следовательно, в силу неравенства (5) центр дискретного нормирования $\nu$ на $V$ есть некоторая точка $x$. Пусть $D_{1}, D_{2} \in \Sigma$ - общие дивизоры, $Z=\left(D_{1} \circ D_{2}\right)$ эффективный алгебраический цикл теоретико-схемного пересечения дивизоров $D_{1}$ и $D_{2}$.

Рассмотрим общую прямую $L \subset \mathbb{P}$. Пусть

$$
\pi_{L}: \mathbb{P}--\rightarrow \mathbb{P}^{M-2}
$$


- соответствующая линейная проекция. Можно считать, что Supp $Z \cap L=\varnothing$, так что $\left.\pi_{L}\right|_{V}$ регулярна в окрестности множества $\operatorname{Supp} Z$. Рассмотрим прямой образ

$$
F=\left(\pi_{L}\right)_{*} Z
$$

Это эффективный дивизор на $\mathbb{P}^{M-2}$. По теореме Лефшшеца кратность каждой компоненты цикла $Z$ не превосходит $n^{2}$, поскольку $Z \equiv n^{2} H^{2}$. Следовательно, можно считать, что это справедливо и для $F$. Очевидно, $\operatorname{deg} F=M n^{2}$.

Следуюший факт является ключевым в наших рассуждениях. Обозначим через $y=\pi_{L}(x)$ образ точки $x$ в $\mathbb{P}^{M-2}$.

ПРЕДЛОЖЕНИЕ 4. Пара $\left(\mathbb{P}^{M-2}, \frac{1}{2 n^{2}} F\right)$

(i) логканонична в точке у в размерности 2,

(ii) не логканонична в точке $y$.

ДокАЗАТЕЛЬСТво утверждения (i) дано в $\S 1$, утверждения (ii) - в $§ 3$ настоящей статьи.

В силу предложения 1 получаем оценку

$$
2+\operatorname{deg}\left(\frac{1}{2 n^{2}} F\right)=2+\frac{M}{2}>M-1,
$$

откуда $M<6$. Противоречие. Следовательно, сделанное вьше предположение о том, что многообразие $V$ не является бирационально сверхжестким, ложно. Теорема доказана.

0.6. Исторические замечания. Как упоминалось вьше, бирациональная сверхжесткость общей гиперповерхности $V=V_{M} \subset \mathbb{P}^{M}$ была доказана в [16]. Позже сверхжесткость была доказана для обших гиперповерхностей с изолированными невырожденными особенностями [21]. В 1998 г. на конференции по алгебраической геометрии в Эссене А. Корти предложил использовать принцип связности Шокурова-Коллара [12], [28] для доказательства бирациональной жесткости. Однако первоначальная версия статьи [2] содержала ошибку, порожденную оптимистичным обобщением принципа связности. Используя ошибочное рассуждение Корти, И. А. Чельцов через несколько месяцев предъявил “доказательство” бирациональной сверхжесткости произвольной гладкой гиперповерхности $V=V_{M} \subset$ $\mathbb{P}^{M}$. Однако оценки, которые он использовал в своих рассуждениях, были слишком сильными, чтобы в их справедливость можно было поверить. В декабре 1998 г. были построены простые контрпримеры к этим оценкам (автором данной статьи и М. М. Гриненко). Попутно была обнаружена ошибка и в первоначальной версии статьи [2]. К сожалению, после исправления этой ошибки оказалось, что новая техника позволяла, главным образом, лишь по-новому доказывать теоремы, уже доказанные классическими методами. В статьях [24], [25] Чельцов доказал сверхжесткость произвольной гладкой гиперповерхности для $M \in\{5,6,7,8\}$, однако некоторые его рассуждения вызывают сомнение (см. подробное изложение в [10]).

В то же время статьи [22], [26], [27] оставляли некоторую надежду на то, что с помощью принципа связности Шокурова-Коллара можно получить новые более сильные оценки для особенностей дивизоров на гиперповерхностях. Косвенно 
эту надежду поддерживало одно рассуждение Корти [2] (значительно упрощенное Чельцовым [26]), исключающее максимальную особенность над трехмерной невырожденной двойной точкой. Эти идеи получили дальнейшее развитие в [3].

В настоящей работе используются и классический метод максимальных особенностей, и принцип связности. Сочетание различных идей позволило, наконец, доказать сверхжесткость гиперповерхностей Фано без досадного предположения об обшности положения (регулярности [16]). Однако для других основных классов многомерных многообразий Фано методы, развитые в настоящей работе, еще недостаточно сильны.

\section{§1. Проекции и кратности}

Цель этого параграфа - доказать утверждение (i) предложения 4. Рассуждения распадаются на две части: сначала доказана оценка для кратности цикла $Z$ вдоль произвольной поверхности, затем эти неравенства распространяются на дивизор $F$ в $\mathbb{P}^{M-2}$.

1.1. Особенности подмногообразий на гиперповерхностях. Сначала рассмотрим следующую общую ситуацию. Пусть $X \subset \mathbb{P}$ - произвольная гиперповерхность, $D$ - эффективный дивизор, высекаемый на $X$ гиперповерхностью степени $n$ в $\mathbb{P}$, иными словами, $D \in|n H|$. В [15], [16] было доказано, что для любой кривой $C \subset X \backslash \operatorname{Sing} X$ выполнена следуюшая оценка:

$$
\operatorname{mult}_{C} D \leqslant n \text {. }
$$

Сформулированное вьше предложение 3 является следствием этого утверждения. Однако оценка (6) есть частный случай следуюшего общего факта, которьй является ключевым для доказательства предложения 4, (i).

ПРЕДЛОЖЕНИЕ 5 . Пусть $W$ - эффективный иикл коразмерности $k, W \sim$ $m H^{k}$ в группе чиклов $A_{M-k-1} X, S \subset W$ - неприводимое подмногообразие размерности $k$, где $2 k+1<M$. Предположим, ито $S \cap \operatorname{Sing} X=\varnothing$. Тогда выполнена следующая оченка:

$$
\operatorname{mult}_{S} W \leqslant m \text {. }
$$

ДоКАЗАТЕЛЬСТво. Используем ту же самую конструкцию, что и в [15]: для неприводимого подмногообразия $T \subset X$ размерности $k, T \cap \operatorname{Sing} X=\varnothing$, и точки $x \in \mathbb{P} \backslash X$ пусть $C(x, T) \subset \mathbb{P}$ - конус с вершиной в точке $x$ и базой $T$. Очевидно,

$$
X \cap C(x, T)=T \cup R(x, T),
$$

где $R(x, T)$ - вычетное замкнутое множество той же размерности, что и $T$. Легко видеть, что для достаточно общей точки $x$ замкнутое множество $R(x, T)$ есть неприводимое подмногообразие и представление (7) справедливо не только в теоретико-множественном, но и в теоретико-схемном смысле.

Пусть теперь $B \subset X$ - любое замкнутое алгебраическое множество. 
Лемма 1. Предположим, что точка $x \in \mathbb{P} \backslash X$ достаточно общая. Тогда:

(i) $e c \wedge u \operatorname{codim}_{X} B>\operatorname{dim} T, m o$

$$
R(x, T) \cap B \subset T \cap B ;
$$

(ii) если $\operatorname{codim}_{X} B=\operatorname{dim} T$, то пересечение $R(x, T) \cap B$ нульмерно вне $T$.

Несложное доказательство леммы дано ниже.

Возьмем обший набор $k$ точек $\left(x_{1}, \ldots, x_{k}\right) \in(\mathbb{P} \backslash X)^{k}$ и построим по индукции по $i=1, \ldots, k$ следуюшую последовательность неприводимых $k$-мерных подмногообразий, начиная с $R_{0}=S$ :

$$
R_{i}=R_{i}\left(x_{1}, \ldots, x_{i}\right)=R\left(x_{i}, R_{i-1}\right) .
$$

Лемма 2. Допустим, что набор $k$ точек $\left(x_{1}, \ldots, x_{k}\right)$ достаточно общий. Тогда пересечение $R_{k} \cap S$ состоит ровно из $\operatorname{deg} R_{k}=(\operatorname{deg} X-1)^{k} \operatorname{deg} S$ различных точек.

Откладывая на некоторое время доказательство леммы, завершим доказательство предложения 5. По лемме 1 пересечение $R_{k} \cap W$ нульмерно, поэтому

$$
m \operatorname{deg} R_{k}=\left(R_{k} \cdot W\right) \geqslant \sum_{p \in R_{k} \cap S}\left(R_{k} \cdot W\right)_{p} \geqslant \operatorname{mult}_{S} W \cdot \operatorname{deg} R_{k} .
$$

Из этой оценки сразу следует предложение 5. Доказательство закончено.

ДокаЗАТЕЛЬСтво лЕммы 1 . Для пары замкнутых множеств $A, B \subset \mathbb{P}$ положим, что

$$
J(A, B)=\overline{\bigcup_{A \ni p \neq q \in B} L_{p, q}}
$$

- замыкание множества, заметаемого прямыми, соединяюшими точки множеств $A$ и $B$. Очевидно,

$$
\operatorname{dim} J(A, B) \leqslant \operatorname{dim} A+\operatorname{dim} B+1 .
$$

Теперь если $\operatorname{codim}_{X} B>\operatorname{dim} T$, то множество $J(T, B)$ имеет положительную коразмерность в $\mathbb{P}$. Если точка $y \in R(x, T) \cap B$ не принадлежит $T$, то, очевидно, $x \in J(T, B)$. Этим часть (i) доказана.

Если $\operatorname{codim}_{X} B=\operatorname{dim} T$, то, вообще говоря, имеет место равенство $J(T, B)=\mathbb{P}$. В этом случае обозначим символом $\mathscr{C} \subset \mathbb{P} \times \mathbb{P} \times \mathbb{P}$ замыкание множества всех коллинеарных троек $(u, v, w)$ с естественными проекциями $\pi_{1}, \pi_{2}, \pi_{3}$ на сомножители $\mathbb{P}$. Имеем

$$
\pi_{3}: \mathscr{C}_{T, B}=\mathscr{C} \cap \pi_{1}^{-1}(T) \cap \pi_{2}^{-1}(B) \rightarrow J(T, B)=\mathbb{P}
$$

- сюръективный морфизм. Поскольку $\operatorname{dim} \mathscr{C}_{T, B}=\operatorname{dim} \mathbb{P}$, он конечен в общей точке. Следовательно, для точки общего положения $x \in \mathbb{P} \backslash X$ прообраз $\left(\left.\pi_{3}\right|_{\mathscr{C}_{T, B}}\right)^{-1}(x)$ есть конечное множество точек. Теперь, как и выше, если точка $y \in R(x, T) \cap B$ не принадлежит множеству $T$, то сушествует точка $z \in T$ такая, что тройка $(z, y, x)$ коллинеарна. Другими словами,

$$
(z, y, x) \in\left(\left.\pi_{3}\right|_{\mathscr{C}_{T, B}}\right)^{-1}(x),
$$

что и требуется доказать. Лемма доказана. 
ДокАЗАТЕЛЬСТво ЛЕммЫ 2. Рассмотрим произвольное неприводимое $k$-мерное подмногообразие $T \subset X$, лежащее вне особого множества, т.е. $T \cap \operatorname{Sing} X=\varnothing$, и вычислим явно пересечение $R(x, T) \cap T$. Пусть

$$
\pi_{x}: X \rightarrow \mathbb{P}^{M-1}
$$

- регулярное отображение, индуцированное линейной проекцией $\mathbb{P}--\rightarrow \mathbb{P}^{M-1}$ из точки $x$. Обозначим символом $D(x) \subset X$ дивизор ветвления морфизма $\pi_{x}$.

Лемма 3. Если точка $x$ достаточно общая, то в теоретико-множественном смысле

$$
R(x, T) \cap T=D(x) \cap T .
$$

ДоКАЗАТЕЛЬСТВо. Напомним, что по предположению $T$ содержится в гладкой части $X$. В произвольной точке $y \in T \backslash D(x)$ дифференциал

$$
\left(d \pi_{x}\right)_{y}: T_{y} X \rightarrow T_{\pi_{x}(y)} \mathbb{P}^{M-1}
$$

есть изоморфизм (так что $\pi_{x}$ есть локальный изоморфизм в комплексно-аналитическом смысле). Принимая во внимание, что отображение $\pi_{x}: T \rightarrow \pi_{x}(T)$ биективно (поскольку по предположению $k<(M-1) / 2$ ), видим, что вблизи точки $y$ прообраз $\pi_{x}^{-1}\left(\pi_{x}(T)\right)$ совпадает с $T$. Отсюда следует, что $y \notin R(x, T)$, так что в теоретико-множественном смысле

$$
R(x, T) \cap T \subset D(x) \cap T .
$$

Покажем, что это включение на самом деле есть равенство. Поскольку точка $x$ общая, можно считать, что $T \cap D^{\circ}(x)$ плотно в $T \cap D(x)$, где $D^{\circ}(x)$ состоит из точек, в которых $\pi_{x}$ имеет простое ветвление, $D^{\circ}(x) \subset D(x)$ - открытое множество. Более того, для подходящего множества локальных параметров $\left(z_{1}, \ldots, z_{M-1}\right)$ в точке $y \in T \cap D^{\circ}(x)$ морфизм $\pi_{x}$ имеет вид

$$
\left(z_{1}, \ldots, z_{M-1}\right) \mapsto\left(z_{1}^{2}, z_{2}, \ldots, z_{M-1}\right)
$$

и можно считать, что $T \neq \pi_{x}^{-1}\left(\pi_{x}(T)\right)$ вблизи точки $y$. В комплексно-аналитическом представлении это означает, что $T$ не является $\sigma$-инвариантным, где

$$
\left(z_{1}, \ldots, z_{M-1}\right) \mapsto\left(-z_{1}, z_{2}, \ldots, z_{M-1}\right)
$$

- локальная инволюция, порожденная двойньм накрытием. Легко видеть, что вблизи точки $y$

$$
T \cap \sigma(T)=T \cap D(x),
$$

где $D(x)=\left\{z_{1}=0\right\}$. Поскольку $\pi_{x}^{-1}\left(\pi_{x}(T)\right)=T \cup \sigma(T)$ в малой комплексно-аналитической окрестности точки $y$, получаем включение

$$
D^{\circ}(x) \cap T \subset R(x, T) \cap T .
$$

Взяв замыкание, получим искомое утверждение. Лемма доказана. 
Отметим, что доказательство проведено на комплексно-аналитическом языке исключительно ради простоты и ясности изложения; доказанный факт устанавливается очевидным образом и на чисто алгебраическом языке.

Теперь рассмотрим систему однородных координат $\left(z_{0}: z_{1}: \ldots: z_{M}\right)$ на $\mathbb{P}$. Пусть $F\left(z_{*}\right)$ - уравнение гиперповерхности $X$. Если $\left(x_{0}: x_{1}: \ldots: x_{M}\right)$ - координаты точки $x$, то

$$
T \cap D(x)=\left\{\left.\sum_{i=0}^{M} \frac{\partial F}{\partial z_{i}} x_{i}\right|_{T}=0\right\}
$$

Поскольку $T \cap \operatorname{Sing} X=\varnothing$, линейная система дивизоров на $T$

$$
\left|\sum_{i=0}^{M} \lambda_{i} \frac{\partial F}{\partial z_{i}}\right|_{T}=0 \mid
$$

не имеет базисных точек. В частности, для достаточно общей точки $x$ дивизор $D(x) \cap T$ неприводим и приведен.

Вернемся теперь к геометрической ситуации, описанной в лемме 2. Напомним, что $k<\operatorname{dim} X / 2$.

Лемма 4. Предположим, что набор $k$ точек $\left(x_{1}, \ldots, x_{k}\right)$ достаточно общий. Тогда для любых $0 \leqslant i<j<l \leqslant k$ имеем

$$
R_{i} \cap R_{l} \subset R_{j}
$$

ДокАЗАтЕльство. Достаточно рассмотреть случай $l=j+1$, но здесь утверждение следует из леммы $1,(\mathrm{i})$, поскольку $R_{l}=R\left(x_{l}, R_{j}\right)$ и $\operatorname{dim} R_{i}=\operatorname{dim} R_{j}=k<$ $\operatorname{dim} X / 2$, что и требовалось доказать.

Итерируя (11) $k$ раз, получаем следуюшее теоретико-множественное равенство:

$$
R_{k} \cap S=\bigcap_{i=0}^{k} R_{i}
$$

Теперь, поскольку по лемме $3 R_{i+1} \cap R_{i}=D\left(x_{i+1}\right) \cap R_{i}$, получаем из равенства (12), что в теоретико-множественном смысле

$$
R_{k} \cap S=D\left(x_{k}\right) \cap \bigcap_{i=0}^{k-1} R_{i}=\cdots=\bigcap_{i=j+1}^{k} D\left(x_{i}\right) \cap \bigcap_{i=0}^{j} R_{i}=\cdots=\bigcap_{i=1}^{k} D\left(x_{i}\right) \cap S .
$$

Однако $\left.D\left(x_{*}\right)\right|_{S}-$ произвольные дивизоры свободной линейной системы. Поэтому пересечение $R_{k} \cap S$ состоит из $\left(D(x)^{k} \cdot S\right)$ различных точек. Остается добавить, что $D(x) \in|(\operatorname{deg} X-1) H|$, где $H$ - класс гиперплоского сечения гиперповерхности $X$. Лемма 2 доказана.

Этим доказательство предложения 5 закончено. 
ЗАмЕчаниЕ 2. Объясним, почему число точек в пересечении $R_{k} \cap S$ оказывается в точности таким, какое необходимо для доказательства нашей оценки. Еще раз рассмотрим конус $C=C(x, T)$. Возьмем разрешение особенностей $\tau: \widetilde{T} \rightarrow T$ и раздуем точку $x$ :

$$
\varphi: \widetilde{\mathbb{P}} \rightarrow \mathbb{P} .
$$

Обозначим символом $\pi: \widetilde{\mathbb{P}} \rightarrow \mathbb{P}^{M-1}$ регуляризованную проекцию из точки $x$ на общую гиперплоскость. Морфизм $\pi$ реализует $\widetilde{\mathbb{P}}$ как локально тривиальное $\mathbb{P}^{1}$-расслоение над $\mathbb{P}^{M-1}$. Возьмем композицию

$$
\beta: \widetilde{T} \stackrel{\tau}{\rightarrow} T \hookrightarrow \widetilde{\mathbb{P}} \stackrel{\pi}{\rightarrow} \mathbb{P}^{M-1}
$$

(где вложение $T \hookrightarrow \widetilde{\mathbb{P}}$ имеет смысл, поскольку $x \notin T$ ) и рассмотрим расслоенное произведение

$$
\widetilde{C}=\widetilde{C}(x, T)=\widetilde{T} \times_{\mathbb{P}^{M-1}} \widetilde{\mathbb{P}} .
$$

Обозначим естественную проекцию на первый сомножитель символом $p: \widetilde{C} \rightarrow \widetilde{T}$. Это отображение есть локально тривиальное $\mathbb{P}^{1}$-расслоение над $\widetilde{T}$. Конечно, образ $\widetilde{C}$ в $\widetilde{\mathbb{P}}$ совпадает с собственным прообразом конуса $C$ на $\widetilde{\mathbb{P}}$. Таким образом, $\widetilde{C}$ получается из $C$ с помошью двух операций: сначала раздувается вершина $x$ конуса, затем разрешение $\tau$ поднимается на соответствуюшее локально тривиальное $\mathbb{P}^{1}$-расслоение над $\pi(T)$. Обозначим это отображение символом

$$
\mu: \widetilde{C} \rightarrow C
$$

Поскольку $\widetilde{C}$ есть $\mathbb{P}^{1}$-расслоение над $\widetilde{T}$, получаем

$$
\operatorname{Pic} \widetilde{C}=\mathbb{Z} E \oplus p^{*} \operatorname{Pic} \widetilde{T}
$$

где $E=\mu^{-1}(x)$ - исключительное сечение, $E \cong \widetilde{T}$. Пусть $T^{\sharp}=\mu^{-1}(T)$ - "базовое" сечение (т. е. происходяшее из исходной базы конуса) и $H^{\sharp}=\mu^{*} H$ - подъем гиперплоского сечения конуса $C \subset \mathbb{P}$. Поскольку и $T^{\sharp}$, и $H^{\sharp}$ суть сечения, из (13) получаем

$$
T^{\sharp} \sim H^{\sharp}+p^{*} Q,
$$

где $Q \in \operatorname{Pic} \widetilde{T}$. Однако, пересекая эти классы с исключительным сечением, получаем равенство

$$
\left.p^{*} Q\right|_{E}=0 .
$$

Тем самым, $Q=0$, потому что композиция

$$
\operatorname{Pic} \widetilde{T} \stackrel{p^{*}}{\rightarrow} \operatorname{Pic} \widetilde{C} \stackrel{\left.\cdot\right|_{E}}{\rightarrow} \operatorname{Pic} E
$$

есть изоморфизм: она индуцирована естественным изоморфизмом $p: E \cong \widetilde{T}$. Равенство (14) получено с учетом того, что, очевидно, и $T \subset C$, и $H \subset C$ (для общего гиперплоского сечения) не содержат вершины конуса, и потому $\left.T^{\sharp}\right|_{E}=\left.H^{\sharp}\right|_{E}=0$. Отсюда заключаем, что

$$
T^{\sharp} \sim H^{\sharp} .
$$


Другими словами, на конусе $C$ дивизор $T=\mu_{*} T^{\sharp}$ рационально эквивалентен гиперплоскому сечению. В частности, выводим из (15), что пересечение $R(x, T) \cap$ $T$ рачионально әквивалентно на $R(x, T)$ гиперплоскому сечению $R(x, T)$.

Это справедливо для любого подмногообразия $T \subset X$. Теперь подсчитаем точки пересечения $R_{k}$ и $S$. Согласно равенству (12) этих точек столько же, сколько точек во множестве

$$
\bigcap_{i=0}^{k} R_{i}=R_{0} \cap R_{1} \cap R_{2} \cap \cdots \cap R_{k} .
$$

Обозначим символом $Q_{j}, j=0,1, \ldots, k$, пересечение $\bigcap_{i=j}^{k} R_{i}$. Поскольку очевидно, что $Q_{j} \subset R_{j}$, получаем из (15), что $Q_{j-1}=R_{j-1} \cap Q_{j}$ рационально әквивалентно на $Q_{j}$ гиперплоскому сечению $Q_{j}$.

Поэтому $Q_{0}=R_{k} \cap S$ рационально эквивалентно пересечению $R_{k}$ с плоскостью $P \subset \mathbb{P}$ коразмерности $k$, т. е. нульмерной схеме степени $\operatorname{deg} R_{k}$. Для доказательства предложения 5 нужен только этот факт, а не точное значение степени $\operatorname{deg} R_{k}$.

Недостаток этого рассуждения состоит в том, что нульмерная схема может содержать кратные точки, так что если строить на нем доказательство, то необходимо проверить, что вклад каждой точки пересечения $R_{k} \cap S$ в оценку (8) правильный (т.е. не меньше, чем ее кратность в схеме $R_{k} \cap S$ ). Конечно, это так и есть, и проверить это не столь сложно, но, тем не менее, проверка требует дополнительных достаточно утомительных рассмотрений. Поэтому прямое вычисление, проведенное вьше, представляется более предпочтительным, а данное рассуждение остается лишь полезным объяснением.

1.2. Изменение кратностей при проекции. Злоупотребляя обозначениями, будем писать $\mathbb{P}$ вместо $\mathbb{P}^{k}$. Пусть $Q \subset \mathbb{P}$ - неприводимое подмногообразие коразмерности $\operatorname{codim} Q \geqslant 2, q \in Q$ - некоторая точка. Для точки $a \in \mathbb{P}$ общего положения морфизм

$$
\pi_{a}: Q \rightarrow R \subset \mathbb{P}^{k-1},
$$

индуцированный линейной проекцией $\pi_{a}: \mathbb{P}--\rightarrow \mathbb{P}^{k-1}$ из точки $a$, бирационален. Положим $r=\pi_{a}(q)$.

ПРЕДЛОЖЕНИЕ 6. Совпадение кратностей

$$
\operatorname{mult}_{r} R=\operatorname{mult}_{q} Q
$$

имеет место тогда и только тогда, когда выполнены следующие два условия:

(i) прямая $L_{a, q}=\overline{\pi_{a}^{-1}\left(\pi_{a}(q)\right)} \subset \mathbb{P}$, соединяющая точки а и q, не имеет других точек пересечения с $Q$ :

$$
L_{a, q} \cap Q=\{q\}
$$

(ii) прямая $L_{a, q}$ не касается $Q$ в точке $q$ :

$$
L_{a, q} \not \subset T_{q} Q .
$$


ДокАЗАТЕЛЬСТво. Для общей плоскости $P \subset \mathbb{P}^{k-1}, P \ni r$, размерности $\operatorname{codim}_{\mathbb{P}} Q-1$ имеем

$$
\operatorname{mult}_{r} R=(P \cdot R)_{r}
$$

Пусть $P^{+}=\overline{\pi_{a}^{-1}(P)}-$ прообраз плоскости $P$ в $\mathbb{P}$. Получаем

$$
(P \cdot R)_{r}=\sum_{y \in \pi_{a}^{-1}(r)}\left(P^{+} \cdot Q\right)_{y}=\left(P^{+} \cdot Q\right)_{q}+\sum_{\substack{y \in \pi_{a}^{-1}(r) \\ y \neq q}}\left(P^{+} \cdot Q\right)_{y}
$$

где $\left(P^{+} \cdot Q\right)_{q} \geqslant$ mult $_{q} Q$. Таким образом, равенство (16) имеет место тогда и только тогда, когда выполнено условие (i) и $\left(P^{+} \cdot Q\right)_{q}=\operatorname{mult}_{q} Q$.

Пусть $\varphi: X \rightarrow \mathbb{P}$ - раздутие точки $q, E \cong \mathbb{P}^{k-1}$ - исключительньй дивизор, $\widetilde{Q}$ и $\widetilde{P}^{+}{ }_{-}$собственные прообразы $Q$ и $P^{+}$на $X$. Легко видеть, что для общей плоскости $P$ пересечение $\widetilde{Q} \cap \widetilde{P}^{+}$нульмерно, так что в силу элементарных формул теории пересечений $[6]$ получаем

$$
\left(P^{+} \cdot Q\right)_{q}=\operatorname{mult}_{q} Q+\sum_{y \in \widetilde{Q} \cap \widetilde{P}^{+} \cap E}\left(\widetilde{Q} \cdot \widetilde{P}^{+}\right)_{y}
$$

Окончательно, равенство (16) вьполнено тогда и только тогда, когда для общей плоскости $P$ пересечение

$$
\widetilde{Q} \cap \widetilde{P}^{+} \cap E
$$

пусто. Но $\widetilde{P}^{+} \cap E$-произвольная плоскость размерности $\operatorname{dim} P$, содержащая точку $\tilde{L}_{a, q} \cap E=T_{q} L_{a, q}$. Следовательно, последнее условие принимает такой вид:

$$
\tilde{L}_{a, q} \cap E \notin \widetilde{Q} \cap E,
$$

т. е. $L_{a, q} \not \subset T_{q} Q$, как и утверждалось. Доказательство закончено.

СлЕДСТвИЕ 1. Если $\operatorname{codim} Q \geqslant 3$, то для любой (возможно, приводимой) кривой $C \subset Q$ проекщия

$$
\pi_{a}: Q \rightarrow R
$$

из достаточно общей точки $a \in \mathbb{P}$ взаимно однозначна и сохраняет кратность в окрестности кривой $C$. Точнее, существует открытое подмножество $U \subset Q$, содержащее кривую $C$ челиком (дополнение $Q \backslash U$ имеет коразмерность 2 в $Q)$, такое, что $\left(\left.\pi_{a}\right|_{Q}\right)^{-1}\left(\pi_{a}(U)\right)=U$, отображение $\pi_{a}: U \rightarrow \pi_{a}(U) \subset R$ биективно и для любой точки $z \in U$ выполнено следующее равенство:

$$
\text { mult }_{\pi_{a}(z)} R=\operatorname{mult}_{z} Q \text {. }
$$


ДокАЗАТЕЛЬСТво. Воспользуемся введенным в п. 1.1 обозначением $J(A, B)$, где $A, B \subset \mathbb{P}$ - любая пара замкнутых подмножеств. Кроме того, для замкнутого множества $A \subset Q$ пусть

$$
T(A, Q)=\overline{\bigcup_{p \in A} T_{p} Q}
$$

обозначает замыкание множества, заметаемого всеми прямыми, касательными к $Q$ в точках множества $A$. Используя оценку (9), видим, что $\operatorname{dim} J(C, Q) \leqslant k-1$, и по этой причине $J(C, Q)$ - собственное замкнутое множество пространства $\mathbb{P}$. Далее, понятно, что

$$
T(C, Q) \subset J(C, Q) .
$$

Следовательно, для точки общего положения

$$
a \in \mathbb{P} \backslash J(C, Q)
$$

любая прямая $L_{a, q}$, где $q \in C$, не имеет других точек пересечения с $Q$ и не касается $Q$ в точке $q$. Поскольку условия (i) и (ii) являются открытыми по $a$ и $q$, сушествует открытое множество $U \subset Q$, содержашее целиком кривую $C$ и удовлетворяюшее (при фиксированной точке $a$ ) условиям (i) и (ii). Следствие доказано.

СлЕДСТВИЕ 2. Предположим, ито $\operatorname{codim} Q=2, C \subset Q-$ кривая (возможно, приводимая) и $q \in C$. При достаточно общем выборе точки $а \in \mathbb{P} \backslash Q$ проекиия

$$
\pi_{a}: Q \rightarrow R \subset \mathbb{P}^{k-1}
$$

биективна и сохраняет кратность в окрестности точки q. Точнее, существует такое открытое множество $U \subset Q$, что:

(i) $C \cap(Q \backslash U)=\Gamma-$ конечное множество точек;

(ii) $\pi_{a}^{-1}\left(\pi_{a}(U)\right)=U$, отображение $\pi_{a}: U \rightarrow \pi_{a}(U)$ биективно и сохраняет кратность;

(iii) для каждой точки $z \in \Gamma$ прямая $L_{a, z}$ не касается $Q$ в $z, L_{a, z} \cap Q=$ $\left\{z, z^{*}\right\}$, где $z^{*} \neq z$ - гладкая точка многообразия $Q$ и $L_{a, z}$ не касается $Q$ в точке $z^{*}$, более того, для каждой точки $z \in \Gamma$ существует такое открытое множество $U_{z} \subset Q, z \in U_{z}$, что для любой точки $v \in U_{z}$ либо прямая $L_{a, v}$ пересекает $Q$ в единственной точке $v$ и при этом пересечение трансверсально, либо $v$ имеет те же свойства, ито и $z$, т. е. прямая $L_{a, v}$ пересекает $Q$ ровно в двух точках $v$ и $v^{*}, v^{*}$ гладкая на $Q$, и в обоих случаях пересечение трансверсально.

ДокАЗАТЕЛЬСтво. В рассматриваемом случае $J(C, Q)=\mathbb{P}$, так что для любой точки $a \in \mathbb{P}$ имеются точки $q \in C$, в которых проекция $\pi_{a}$ увеличивает кратность. Однако если $A \subset C$ - произвольное конечное множество точек, то $\operatorname{dim} J(A, Q) \leqslant k-1$. Следовательно, для общей точки $a \in \mathbb{P}$ существует такое открытое множество $U \subset Q$, что выполнены условия (i) и (ii). (Для того чтобы получить условие (i), достаточно выбрать множество $A$ таким образом, чтобы оно содержало по крайней мере одну точку $a_{i} \in A, a_{i} \in C_{i}$, на каждой неприводимой компоненте $C_{i}$ кривой $C$.) 
Далее, $\operatorname{dim} J(C, \operatorname{Sing} Q) \leqslant k-1$, так что для каждой точки $z \in \Gamma$ прямая $L_{a, z}$ пересекает $Q$ (кроме самой точки $z$ ) только в гладких точках. Подсчитьвая размерности, легко увидеть, что для общей точки $a$ пересечение $L_{a, z}$ с $Q$ состоит ровно из двух точек $z, z^{*}$, причем в точке $z^{*}$ это пересечение трансверсально. Наконец, легко видеть, что свойство прямой $L_{a, v}$ пересекать $Q$ трансверсально не более чем в двух точках (в самой $v$ и, возможно, еще в одной $v^{*}$, гладкой на $Q$ ) является открытым по $v$. Это доказывает последнее утверждение. Доказательство закончено.

Теперь легко обобщить следствия 1 и 2 на случай эффективных алгебраических циклов (вместо неприводимых подмногообразий). Пусть

$$
Q=\sum_{i \in I_{Q}} m_{i} Q_{i}
$$

- эффективный цикл, где $Q_{i}$ попарно различны и имеют одинаковую размерность $\operatorname{dim} Q_{i}=\operatorname{dim} Q, m_{i} \geqslant 1$. Кратность цикла $Q$ в точке $y \in \mathbb{P}$ определим по линейности:

$$
\text { mult }_{y} Q=\sum_{i \in I_{Q}} m_{i} \text { mult }_{y} Q_{i}
$$

Легко видеть, что утверждение следствия 1 остается верным, если заменить неприводимое подмногообразие $Q$ эффективным циклом $Q$, а подмногообразие $R=$ $\pi_{a}(Q)$ - циклом

$$
R=\left(\pi_{a}\right)_{*} Q=\sum_{i \in I_{Q}} m_{i} R_{i},
$$

где $R_{i}=\left(\pi_{a}\right)_{*} Q_{i}=\pi_{a}\left(Q_{i}\right)$, и можно снова считать, что подмногообразия $R_{i}$ попарно различны.

Утверждения (i) и (ii) следствия 2 также остаются верными после этой замены. Утверждение (iii) сформулируем следуюшим образом:

(iii) для каждой точки $z \in \Gamma$ прямая $L_{a, z}$ не касается носителя $\operatorname{Supp} Q$ в $z, \quad L_{a, z} \cap \operatorname{Supp} Q=\left\{z, z^{*}\right\}$, әде $z^{*} \neq z-$ гладкая точка замкнутого множества $\operatorname{Supp} Q$, и $L_{a, z}$ не касается $\operatorname{Supp} Q$ в точке $z^{*}$, другими словами, существует одна и только одна компонента $Q_{i(z)}$ чикла $Q, i(z) \in I_{Q}$, такая, что $Q_{i(z)} \ni z^{*}$ и $Q_{i(z)}$ гладкая в точке $z^{*} ;$ в частности,

$$
\operatorname{mult}_{\pi_{a}(z)} R=\operatorname{mult}_{z} Q+m_{i(z)} \leqslant \operatorname{mult}_{z} Q+\max _{i \in I_{Q}}\left\{m_{i}\right\},
$$

более того, для каждой точки $z \in \Gamma$ существует такое открытое множество $U_{z} \subset \operatorname{Supp} Q, \quad z \in U_{z}$, что для любой точки $v \in U_{z}$ либо прямая $L_{a, v}$ пересекает $\operatorname{Supp} Q$ только в точке $v$ и это пересечение трансверсально, либо $v$ обладает теми же свойствами, что и $z$, т. е. прямая $L_{a, v}$ пересекаem $\operatorname{Supp} Q$ ровно в двух точках $v$ и $v^{*}, v^{*}$ гладкая на $\operatorname{Supp} Q$, и в обоих случаях пересечение трансверсально; в частности, для любой точки $v \in U_{z}$ имеет место оценка

$$
\operatorname{mult}_{\pi_{a}(v)} R \leqslant \operatorname{mult}_{v} Q+\max _{i \in I_{Q}}\left\{m_{i}\right\}
$$


ДоКАЗАТЕЛЬСТВО УТВЕРЖДЕНИЯ (iii). Кроме оценки (17), все перечисленное вытекает непосредственно из п. (iii) следствия 2. Поэтому докажем эту оценку. Для общей 2-плоскости $P \subset \mathbb{P}^{k-1}$, содержащей точку $r=\pi_{a}(z)$, имеем

$$
\operatorname{mult}_{r} R=(P \cdot R)_{r}
$$

Как и в доказательстве предложения 6 , возьмем прообраз $P^{+}=\overline{\pi_{a}^{-1}(P)}$ плоскости $P$ и получим равенство

$$
(P \cdot R)_{r}=\left(P^{+} \cdot Q\right)_{z}+\left(P^{+} \cdot Q\right)_{z^{*}}
$$

Поскольку прямая $L_{a, z}$ не касается $\operatorname{Supp} Q$ в точках $z$ и $z^{*}$ и поскольку $z^{*}$ - гладкая точка подмногообразия $Q_{i(z)}$, можно переписать $(19)$, как левую часть неравенства (17). Остальное очевидно. Доказательство этим завершено.

В дальнейшем, ссылаясь на следствия 1 и 2 , подразумеваем их обобшенную форму, которую обсуждали выше (относящуюся к равноразмерным циклам $Q$ ).

1.3. Особенности дивизора $F$. Докажем часть (i) предложения 4. Отметим, что если подмногообразие $W \subset X$ гладкого многообразия $X$ есть логцентр пары $(X, D)$, где $D=\sum_{i \in I_{D}} d_{i} D_{i}-\mathbb{Q}$-дивизор, то для любой точки $x \in W$ выполнено неравенство

$$
\operatorname{mult}_{x} D=\sum_{i \in I_{D}} d_{i} \operatorname{mult}_{x} D_{i}>1
$$

Следовательно, если точка $x \in \mathbb{P}^{M-2}$ лежит на логцентре пары $\left(\mathbb{P}^{M-2}, \frac{1}{2 n^{2}} F\right)$, то

$$
\operatorname{mult}_{x} F>2 n^{2} \text {. }
$$

Обозначим символом $C \ni x$ связную компоненту множества

$$
\left\{z \in Z \mid \operatorname{mult}_{x} Z>n^{2}\right\},
$$

содержащую точку $x$. Поскольку $Z \sim n^{2} H^{2}$ - эффективный цикл коразмерности два на гладкой гиперповерхности $V$, можно в силу предложения 5 сделать вывод, что $C$ есть либо точка, либо кривая.

Представим проекцию из прямой $L$ в виде композиции двух проекций:

$$
\pi_{L}=\pi_{a} \circ \pi_{b},
$$

где $b \in \mathbb{P}$ и $a \in \mathbb{P}^{M-1}$ - точки общего положения. Положим $Z_{b}=\left(\pi_{b}\right)_{*} Z$. Согласно следствию 1 в Supp $Z$ существует такое открытое подмножество $U_{b} \supset C$, что $\left.\pi_{b}\right|_{U_{b}}$ сохраняет кратности. Следовательно, связное замкнутое алгебраическое множество $C_{b}=\pi_{b}(C)$ есть связная компонента множества

$$
\left\{z \in \operatorname{Supp} Z_{b} \mid \operatorname{mult}_{z} Z_{b}>n^{2}\right\} .
$$


Если $C=\{x\}$ - точка, то $C_{b}=\pi_{b}(x)$ - тоже точка, и, применяя снова следствие 1 , заключаем, что для любой точки $z$ в некоторой окрестности точки $y$ прообраз $\left(\left.\pi_{L}\right|_{\operatorname{Supp}} z\right)^{-1}(z)$ состоит из единственной точки, и если $z \neq y=\pi_{a}\left(\pi_{b}(x)\right)=$ $\pi_{L}(x)$, то имеет место оценка

$$
\operatorname{mult}_{z} F=\operatorname{mult}_{\left(\left.\pi_{L}\right|_{\operatorname{Supp} Z}\right)^{-1}(z)} Z \leqslant n^{2}
$$

так что пара $\left(\mathbb{P}^{M-2}, \frac{1}{2 n^{2}} F\right)$ логтерминальна в точке $y$ в размерности 1.

Поэтому считаем, что $C$ - связная кривая. Тем самым, $C_{b} \subset \operatorname{Supp} Z_{b}$ - также связная кривая и можно применить следствие 2 (в обобщенной форме) к эффективному циклу $Z_{b}$ в окрестности кривой $C_{b}$. Получаем, что существует открытое подмножество $U_{a} \subset \operatorname{Supp} Z_{b}$, содержащее точку $\pi_{b}(x)$ и пересекающее каждую компоненту кривой $C_{b}$, такое, что отображение $\pi_{a}: U_{a} \rightarrow \pi_{a}\left(U_{a}\right) \subset \pi_{L}(\operatorname{Supp} Z)$ взаимно однозначно и сохраняет кратность. В частности,

$$
\pi_{L}(C) \cap \pi_{a}\left(U_{a}\right)=\left\{z \in \pi_{a}\left(U_{a}\right) \mid \operatorname{mult}_{z} F>n^{2}\right\}
$$

Поэтому никакое многообразие $W \subset \operatorname{Supp} F$, имеюшее непустое пересечение с $\pi_{a}\left(U_{a}\right)$ и не содержашееся целиком в $\pi_{L}(C)$, не может быть логцентром пары $\left(\mathbb{P}^{M-2}, \frac{1}{2 n^{2}} F\right)$.

Остается рассмотреть любую из “плохих" точек $p \in \Gamma=C_{b} \cap\left(\operatorname{Supp} Z_{b} \backslash U_{a}\right)$. Прямая $L_{a, p}$ не касается $Z_{b}$ в точке $p$ и

$$
L_{a, p} \cap \operatorname{Supp} Z_{b}=\left\{p, p^{*}\right\},
$$

где $p^{*} \neq p$ - гладкая точка одной из неприводимых компонент цикла $Z_{b}$, и, более того, пересечение $L_{a, p}$ и $\operatorname{Supp} Z_{b}$ в точке $p^{*}$ трансверсально. В силу п. (iii) следствия 2 (в обобщенной форме) получаем, что существует такая окрестность $U_{p} \ni p$, что для любой точки $v \in U_{p}$ выполнена оценка (18). Поскольку кратность каждой неприводимой компоненты цикла $Z$ не превосходит $n^{2}$ (по теореме Лефшеца) и, соответственно, то же самое верно для $Z_{b}$, получаем окончательно для любой точки $q \in U_{p}:$

$$
\operatorname{mult}_{\pi_{a}(q)} F \leqslant \operatorname{mult}_{q} Z_{b}+n^{2}
$$

Отметим, что $C_{b}-$ связная компонента множества $\left\{z \in Z_{b} \mid \operatorname{mult}_{z} Z_{b}>n^{2}\right\}$. B силу (20) отсюда следует, что в некоторой окрестности точки $\pi_{a}(p)$ связное множество $\pi_{a}\left(C_{b}\right)$ содержит все точки $z \in F$, удовлетворяющие неравенству

$$
\operatorname{mult}_{z} F \geqslant 2 n^{2}+1
$$

Следовательно, кривая $\pi_{a}\left(C_{b}\right)$ имеет такую окрестность $U \subset \operatorname{Supp} F, U \supset \pi_{a}\left(C_{b}\right)$, что

$$
\pi_{a}\left(C_{b}\right) \supset\left\{z \in U \mid \text { mult }_{z} F \geqslant 2 n^{2}+1\right\} .
$$

Поэтому пара $\left(\mathbb{P}^{M-2}, \frac{1}{2 n^{2}} F\right)$ логканонична в точке $y=\pi_{L}(x)$ в размерности 2 . 


\section{§2. Логканонические особенности}

В этом параграфе доказано предложение 1. Рассуждения основаны на принципе связности Шокурова-Коллара.

2.1. Принцип связности Шокурова-Коллара. Нам понадобится частный случай этого принципа. Пусть $X$ - гладкое многообразие, $D=\sum_{i \in I_{D}} d_{i} D_{i}-$ $\mathbb{Q}$-дивизор, $d_{i}>0$, где простые дивизоры $D_{i}$ попарно различны. Пусть $f: Y \rightarrow X-$ разрешение особенностей, $\left\{E_{i} \mid i \in I_{f}\right\}$ - множество исключительных дивизоров, причем дивизор

$$
\bigcup_{i \in I_{D}} \widetilde{D}_{i} \cup \bigcup_{i \in I_{f}} E_{i}
$$

имеет нормальные пересечения на $Y, \widetilde{D}_{i}$ обозначает собственный прообраз $D_{i}$ на $Y$. Положим

$$
\widetilde{D}=\sum_{i \in I_{D}} d_{i} \widetilde{D}_{i}
$$

и запишем

$$
K_{Y}+\widetilde{D}=f^{*}\left(K_{X}+D\right)+\sum_{i \in I_{f}} e_{i} E_{i} .
$$

ПринциП Связности [28], [12]. Предположим, что класс $-\left(K_{X}+D\right)$ численно эффективен и обтемен. Тогда замкнутое алгебраическое мнохество

$$
\bigcup_{d_{i} \geqslant 1} D_{i} \cup f\left(\bigcup_{e_{i} \leqslant-1} E_{i}\right)
$$

связно.

В основе доказательства (извлеченного из [12, п. 17.4]) лежит

ТЕОРемА КАВАМАТЫ-ФИВЕГА (об обрашении в нуль). Пусть $Y$ - гладкое проективное многообразие, $R=\sum_{i \in I_{R}} r_{i} R_{i}$ - әффективный $\mathbb{Q}$-дивизор с коэффиииентами $0<r_{i}<1$, әде $R_{i}$ попарно различны и дивизор

$$
\bigcup_{i \in I_{R}} R_{i}
$$

имеет нормальные пересечения. Предположим, что дивизор $L \in \operatorname{Pic} Y$ maков, что класс $(L-R)$ численно әффективен и обвемен, т. е. $(L-R)^{\operatorname{dim} Y}>0$. Тогда

$$
H^{j}\left(Y, K_{Y}+L\right)=0
$$

для $j \geqslant 1$.

Доказательство, обобщения и пояснения см. в [5], [13], [29].

Теперь, следуя работам А. Коллара, докажем принцип связности. Для удобства обозначений положим

$$
J=I_{f} \cup I_{D}
$$


и при $i \in I_{D}$ положим $E_{i}=\widetilde{D}_{i}, e_{i}=-d_{i}$. Теперь можно переписать $(21)$ как

$$
K_{Y}=f^{*}\left(K_{X}+D\right)+\sum_{i \in J} e_{i} E_{i}
$$

Для каждого $i \in J$ положим

$$
m_{i}=\min \left\{m \in \mathbb{Z} \mid m \geqslant e_{i}\right\}, \quad \alpha_{i}=m_{i}-e_{i} \geqslant 0,
$$

так что $e_{i}=m_{i}-\alpha_{i}, \alpha_{i}<1$. Перепишем $(22)$ в следующем виде:

$$
-f^{*}\left(K_{X}+D\right)=-K_{Y}+\sum_{i \in J} m_{i} E_{i}-\sum_{i \in J} \alpha_{i} E_{i}
$$

Поскольку по предположению класс $-\left(K_{X}+D\right)$ численно эффективен и объемен, то же самое верно и для его $f$-подъема. Теперь применим теорему об обращении в нуль для

$$
L=-K_{Y}+\sum_{i \in J} m_{i} E_{i} \quad \text { и } \quad R=\sum_{i \in J} \alpha_{i} E_{i} .
$$

Получим обращение в нуль когомологий

$$
H^{j}\left(Y, \sum_{i \in J} m_{i} E_{i}\right)=0
$$

для $j \geqslant 1$. С другой стороны, $m_{i} \in \mathbb{Z}_{+}$тогда и только тогда, когда $e_{i}>-1$, и, более того, $m_{i} \geqslant 1$ тогда и только тогда, когда $e_{i}>0$. Поэтому, полагая

$$
E^{+}=\sum_{e_{i}>-1} m_{i} E_{i}=\sum_{e_{i}>0} m_{i} E_{i}
$$

и

$$
E^{-}=-\sum_{e_{i} \leqslant-1} m_{i} E_{i}
$$

видим, что

$$
H^{j}\left(Y, E^{+}-E^{-}\right)=0
$$

для $j \geqslant 1$, где оба дивизора $E^{+}, E^{-}$эффективны. Теперь применим $(23)$ к стандартной точной последовательности

$$
\left.0 \rightarrow \mathscr{O}_{Y}\left(E^{+}-E^{-}\right) \rightarrow \mathscr{O}_{Y}\left(E^{+}\right) \rightarrow \mathscr{O}_{Y}\left(E^{+}\right)\right|_{E^{-}} \rightarrow 0
$$

Получаем, что отображение

$$
H^{0}\left(Y, E^{+}\right) \rightarrow H^{0}\left(E^{-},\left.E^{+}\right|_{E^{-}}\right)
$$

сюръективно. Но поскольку для всех $i \in I_{D}$ коэффициенты $e_{i}$ отрицательны, дивизор $E^{+}$является $f$-исключительным. Поэтому

$$
H^{0}\left(Y, E^{+}\right) \cong \mathbb{C}
$$


С другой стороны, компоненты $E_{i}$ дивизоров $E^{+}$и $E^{-}$образуют два непересекающихся множества, так что ограничение $\left.E^{+}\right|_{E^{-}}$есть эффективньй дивизор на схеме $E^{-}$.

Пусть $l \geqslant 1$ - число связных компонент множества

$$
\bigcup_{e_{i} \leqslant-1} E_{i}
$$

которое есть в точности носитель $\operatorname{Supp} E^{-}$. Очевидно, $\operatorname{dim} H^{0}\left(E^{-},\left.E^{+}\right|_{E^{-}}\right) \geqslant l$. Поэтому (24) дает сюръективное отображение

$$
\mathbb{C} \rightarrow \mathbb{C}^{l} \rightarrow 0,
$$

откуда, конечно, следует, что $l \leqslant 1$. Другими словами, множество Supp $E^{-}$связно. Именно это и утверждалось. Принцип связности доказан.

2.2. Логканонические особенности дивизоров в $\mathbb{P}^{k}$. Докажем предложение 1. Достаточно установить “терминальньй” вариант этого предложения, поскольку "канонический" вариант из него немедленно следует. Более того, поскольку свойство логтерминальности является открытым относительно коэффициентов $d_{i}$, можно считать, что неравенство (3) строгое, т. е.

$$
l+\operatorname{deg} D<k+1 .
$$

Теперь допустим противное:

$$
x \in \mathrm{LC}(X, D) .
$$

По предположению $\operatorname{dim} \operatorname{LC}(X, D, x) \leqslant l-1$, так что для общей плоскости $P \subset \mathbb{P}^{k}$ коразмерности $l$ имеем

$$
P \cap \operatorname{LC}(X, D, x)=\varnothing .
$$

Для упрошения обозначений пишем в этом пункте $\mathbb{P}$ вместо $\mathbb{P}^{k}$.

Зафиксируем такую плоскость $P$. Обозначим символом $\Lambda_{P} \subset|H|$ линейную систему гиперплоскостей, содержащих $P$. Если $l=1$, то $\Lambda_{P}=\{P\}$. В этом случае положим $H^{\sharp}=P$.

Если $l \geqslant 2$, то линейная система $\Lambda_{P}$ не имеет неподвижных компонент, Bs $\Lambda_{P}=$ $P$. В этом случае положим

$$
H^{\sharp}=\sum_{i \in I_{H}} \varepsilon H_{i},
$$

где $\varepsilon>0$ - малое рациональное число, $\left\{H_{i} \mid i \in I_{H}\right\}$ - общий набор гиперплоскостей в $\Lambda_{P}$ и

$$
\operatorname{deg} H^{\sharp}=l=\varepsilon \cdot \sharp I_{H} .
$$

Рассмотрим новый $\mathbb{Q}$-дивизор $D^{\sharp}=D+H^{\sharp}$.

Лемма 5. Вне плоскости $P$ замкнутые множества $\operatorname{LC}(\mathbb{P}, D)$ и $\operatorname{LC}\left(\mathbb{P}, D^{\sharp}\right)$ совпадают. 
ДоКАЗАТЕЛЬСТво. Это почти очевидно. Пусть $f: Y \rightarrow \mathbb{P}$ - разрешение пары $(\mathbb{P}, D)$. Ясно, что вне $P$ дивизор $H^{\sharp}$ имеет нормальные пересечения и

$$
\left.\widetilde{H}^{\sharp}\right|_{Y \backslash f^{-1}(P)}=\left.f^{-1}\left(H^{\sharp}\right)\right|_{Y \backslash f^{-1}(P)},
$$

так что на $Y \backslash f^{-1}(P)$ получаем

$$
K_{Y}+\widetilde{D}^{\sharp}=f^{*}\left(K_{\mathbb{P}}+D^{\sharp}\right)+\sum_{i \in I_{f}} e_{i} E_{i} .
$$

Более того, поскольку на $Y \backslash f^{-1}(P)$ линейная система $f^{*} \Lambda_{P}$ свободна, дивизор

$$
\bigcup_{i \in I_{D}} \widetilde{D}_{i} \cup \bigcup_{i \in I_{f}} E_{i} \cup \bigcup_{i \in I_{H}} \widetilde{H}_{i}
$$

имеет на $Y \backslash f^{-1}(P)$ нормальные пересечения. Принимая во внимание, что $\varepsilon>0$ мало, окончательно получаем

$$
\mathrm{LC}\left(\mathbb{P} \backslash P, D^{\sharp}\right)=f\left(\bigcup_{e_{i} \leqslant-1} E_{i}\right)=\mathrm{LC}(\mathbb{P} \backslash P, D),
$$

что и требуется доказать.

Теперь для некоторого разрешения $f: Y \rightarrow \mathbb{P}$ пары $\left(\mathbb{P}, D^{\sharp}\right)$ запишем

$$
K_{Y}=f^{*}\left(K_{\mathbb{P}}+D^{\sharp}\right)-\sum_{i \in I_{D}} d_{i} \widetilde{D}_{i}-\sum_{i \in I_{H}} \varepsilon \widetilde{H}_{i}+\sum_{i \in I_{f}^{*}} e_{i} E_{i}+e_{P} E,
$$

где при $l=1$ полагаем $\varepsilon=0, E=\widetilde{P}$ и $e_{P}=-1$, а при $l \geqslant 2$ исключительный дивизор $E$ определен следуюшим условием: $f(E)=P$ и в некоторой окрестности общей точки дивизора $E$ морфизм $f: Y \rightarrow \mathbb{P}$ есть раздутие плоскости $P$.

Лемма 6. Имеет место равенство е ${ }_{P}=-1$.

ДоказАТЕльство. В самом деле, $\sum_{i \in I_{H}} \varepsilon=l$, в то время как дискрепантность дивизора $E$ равна $a(E)=\operatorname{codim} P-1=l-1$, откуда и получаем утверждение леммы.

Отметим, что $-\left(K_{\mathbb{P}}+D^{\sharp}\right)$ - численно эффективньй и объемньй класс, точнее, он равен $(k+1-l-\operatorname{deg} D) H$, где коэффициент, стояший в скобках, положительньй. Теперь в силу принципа связности и двух предыдущих лемм множество

$$
f\left(\bigcup_{e_{i} \leqslant-1} E_{i}\right) \cup P=\operatorname{LC}(\mathbb{P}, D) \cup P
$$

связно. Однако связная компонента $\mathrm{LC}(\mathbb{P}, D, x)$ по предположению непуста и, более того,

$$
\mathrm{LC}(\mathbb{P}, D, x) \cap P=\varnothing .
$$

Поэтому замкнутое множество $\mathrm{LC}(\mathbb{P}, D) \cup P$ имеет по крайней мере две связные компоненты. Противоречие. Предложение 1 доказано. 


\section{§3. Основная конструкция}

В этом параграфе докажем утверждение (ii) предложения 4. В основе рассуждений лежит геометрическая конструкция, которая была введена в [15].

\section{1. Прямой образ максимальной особенности. Пусть}

$$
\pi_{L}: \mathbb{P}--\rightarrow \mathbb{P}^{M-2}
$$

- линейная проекция из общей прямой $L$,

$$
\left.\pi_{L}\right|_{V}: V--\rightarrow \mathbb{P}^{M-2}
$$

- ее ограничение на гиперповерхность $V$. Отображение $\left.\pi_{L}\right|_{V}$ не определено в $M$ точках пересечения $L \cap V$. Раздуем эти $M$ точек:

$$
\sigma: \widetilde{V} \rightarrow V
$$

и обозначим регулярное продолжение отображения $\left.\pi_{L}\right|_{V}$ на $\widetilde{V}$ символом

$$
\pi: \widetilde{V} \rightarrow \mathbb{P}^{M-2} .
$$

Если прямая $L$ достаточно общая, то выполнены следующие условия:

1) $L \cap \operatorname{Supp} Z=\varnothing$, так что для собственного прообраза $\widetilde{Z}$ эффективного цикла $Z=\left(D_{1} \circ D_{2}\right)$ на $\widetilde{V}$ имеет место равенство $\widetilde{Z}=\sigma^{-1}(Z)$;

2 ) кривая $\pi^{-1}(y)$ гладкая (напомним, что $y=\pi_{L}(x)$ ), она пересекает $Z$ в единственной точке $x$ и не касается $Z$ в $x$;

3 ) морфизм $\pi$ бирационален на каждой неприводимой компоненте цикла $Z$.

Рассмотрим прямой образ $\pi_{*} \nu$ дискретного нормирования $\nu$. Алгебраически $\pi_{*} \nu$ можно определить (с точностью до деления на некоторое целое число) как композицию

$$
\pi_{*} \nu: \mathbb{C}\left(\mathbb{P}^{M-2}\right)^{\times} \stackrel{\pi^{*}}{\rightarrow} \mathbb{C}(\widetilde{V})^{\times}=\mathbb{C}(V)^{\times} \stackrel{\nu}{\rightarrow} \mathbb{Z},
$$

где верхний индекс ${ }^{\times}$означает, что берется мультипликативная группа ненулевых элементов поля. Реализуем нормирование $\pi_{*} \nu$ геометрически. Пусть

$$
\varphi: X \rightarrow \mathbb{P}^{M-2}
$$

- бирациональный морфизм, где многообразие $X$ гладкое, $\left\{E_{i} \mid i \in I_{X}\right\}$ - множество исключительных дивизоров, имеющее нормальные пересечения, и

$$
\varphi\left(\bigcup_{i \in I_{X}} E_{i}\right)=y
$$

причем для некоторого исключительного дивизора $E=E_{a}$ имеем

$$
\pi_{*} \nu=\operatorname{ord}_{E}(\cdot)
$$


Геометрический смысл этой операции состоит в следующем. Рассмотрим расслоенное произведение $V^{*}=\widetilde{V} \times_{\mathbb{P} M-2} X$. Принимая во внимание $(25)$ и тот факт, что слой $\pi^{-1}(y)$ неособый, видим, что многообразие $V^{*}$ гладкое и проекция $f: V^{*} \rightarrow \widetilde{V}$ есть бирациональный морфизм с множеством исключительных дивизоров $\left\{E_{i}^{*} \mid i \in I_{X}\right\}$, где, более того,

$$
E_{i}^{*}=C \times E_{i} \quad \text { и } f\left(\bigcup_{i \in I_{X}} E_{i}^{*}\right)=C,
$$

причем $\left.f\right|_{E_{i}^{*}}-$ проекция на первый сомножитель. Далее, коммутативна следующая диаграмма:

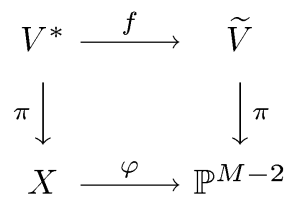

где, несколько злоупотребляя нашими обозначениями, обозначаем естественную проекцию многообразия $V^{*}$ на $X$ тем же символом $\pi$, поскольку вне $\bigcup E_{i}^{*}$ многообразие $V^{*}$ изоморфно $\widetilde{V}$ и левая проекция продолжает правую.

Теперь по определению прямого образа получаем

$$
\pi\left(\operatorname{centre}\left(\nu, V^{*}\right)\right)=E
$$

Поскольку centre $(\nu, \widetilde{V})=x$, можно сказать больше. Для каждого $i \in I_{X}$ положим

$$
\Delta_{i}=E_{i}^{*} \cap f^{-1}(x) \subset E_{i}^{*} .
$$

Все проекции $\pi: \Delta_{i} \rightarrow E_{i}$ суть изоморфизмы. Теперь получаем

$$
\operatorname{centre}\left(\nu, V^{*}\right)=\Delta
$$

где $\Delta=\Delta_{a} \subset E^{*}=E_{a}^{*}$.

Пусть $\Sigma^{*}$ - собственный прообраз линейной системы $\Sigma$ на $V^{*}$.

ЛЕмма 7. Собственный прообраз совпадает с полным прообразом:

$$
\Sigma^{*}=f^{*} \widetilde{\Sigma}=f^{*} \sigma^{*} \Sigma
$$

ДоКАЗАТЕЛЬСТВо. Как упоминалось вьше, для каждого исключительного дивизора $E_{i}^{*}$ имеем равенство $f\left(E_{i}^{*}\right)=C$, но кривая $C$ не является базисной для $\widetilde{\Sigma}$, поскольку $C \not \subset \widetilde{Z}$. Лемма доказана. 
3.2. Сравнение кратностей. Ниже используются кольцевая структура на группе классов циклов на гладких многообразиях и операции прямого образа и плоского обратного образа, описанные в [6].

Рассмотрим дивизоры

$$
D_{i}^{*}=f^{*} \widetilde{D}_{i}=f^{*} \sigma^{*} D_{i}, \quad i=1,2
$$

Пусть

$$
Z^{*}=\left(D_{1}^{*} \circ D_{2}^{*}\right), \quad \widetilde{Z}=\left(\widetilde{D}_{1} \circ \widetilde{D}_{2}\right)
$$

- алгебраические циклы теоретико-схемного пересечения этих дивизоров. Обозначим символами $z^{*}, \widetilde{z}$ и $z$ классы циклов $Z^{*}, \widetilde{Z}$ и $Z$ в $A^{2} V^{*}, A^{2} \widetilde{V}$ и $A^{2} V$ соответственно.

СлеДСТВИЕ 3 (из леммы 7). Имеет место равенство $z^{*}=f^{*} \widetilde{z}=f^{*} \sigma^{*} z$.

ДокАЗАТЕЛЬСТво. В самом деле, $f^{*}$ и $f^{*} \sigma^{*}$-кольцевые гомоморфизмы. Следствие доказано.

Теперь сравним прямой образ $F$ цикла $Z$ на $\mathbb{P}^{M-2}$ и прямой образ цикла $Z^{*}$ на $X$.

Лемма 8. Выполнено равенство $\pi_{*} Z^{*}=\varphi^{*} F$.

ДокАЗАТЕльСТво. По построению $\pi_{*} Z=F$, следовательно, $\pi_{*} \widetilde{Z}=F$. Отсюда получаем

$$
\pi_{*} Z^{*}=\widetilde{F}+\sum_{i \in I_{X}} b_{i} E_{i}
$$

где $\widetilde{F}$ - собственный прообраз цикла $F$ на $X, b_{i} \in \mathbb{Z}$ - однозначно определенные целые числа. С другой стороны,

$$
\varphi^{*} F=\widetilde{F}+\sum_{i \in I_{X}} c_{i} E_{i}
$$

где $c_{i}=\operatorname{ord}_{E_{i}} F \in \mathbb{Z}_{+}$. Однако в силу предыдущего следствия (и стандартных теорем теории пересечений $[6$, п. 1.7 и гл. 8]) имеем

$$
\pi_{*} z^{*}=\pi_{*}\left(f^{*} \sigma^{*} z\right)=\varphi^{*} \pi_{*} \widetilde{z}
$$

(здесь $f^{*}, \varphi^{*}$ и $\sigma^{*}$ - операции подъема в $A^{*}, \pi_{*}$ - операция прямого образа, сохраняющая размерность), так что классы двух дивизоров

$$
\sum_{i \in I_{X}} b_{i} E_{i} \quad \text { и } \quad \sum_{i \in I_{X}} c_{i} E_{i}
$$

в $A^{1} X$ совпадают. Следовательно, $b_{i}=c_{i}$ для всех $i \in I_{X}$, что и доказывает лемму. 
СлЕДСТВИЕ 4. Выполнено следующее равенство:

$$
\nu_{E}(F)=\text { mult }_{\Delta} Z^{*}
$$

Здесь правая часть обозначает кратность, с которой неприводимое подмногообразие $\Delta \subset V^{*}$ коразмерности два входит в цикл $Z^{*}$.

3.3. Вычисление кратности $\nu_{E}(F)$. Правую часть равенства (26) вычислить нетрудно (фактически это уже было сделано в [15]). Именно, пусть

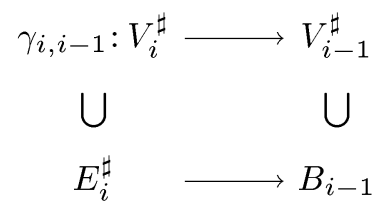

- последовательность раздутий центров нормирования $\nu$, начинающаяся с $V_{0}^{\sharp}=$ $V^{*}, B_{0}=\Delta$, т. е. $B_{i}=\operatorname{centre}\left(\nu, V_{i}^{\sharp}\right), E_{i}^{\sharp}=\gamma_{i, i-1}^{-1}\left(B_{i-1}\right), i=1, \ldots, N, E_{N}^{\sharp} \subset V_{N}^{\sharp}-$ дивизориальная реализация нормирования $\nu$.

Пусть $\Sigma^{i}-$ собственный прообраз линейной системы $\Sigma$ на $V_{i}^{\sharp}$. Определим кратности $\mu_{i}$ формулой

$$
\mu_{i}=\operatorname{mult}_{B_{i-1}} \Sigma^{i-1} .
$$

Построим обычным образом (см. [1], [11], [14]-[21]) граф Г исключительных дивизоров $E_{i}^{\sharp}, i=1, \ldots, N$. Пусть

$$
\left\{E_{i}^{\sharp}\right\} \leftrightarrow\{i=1, \ldots, N\}
$$

- множество вершин, и ориентированное ребро (стрелка)

$$
i \rightarrow j
$$

соединяет две вершины тогда и только тогда, когда $i>j$ и подмногообразие $B_{i-1}$ содержится в собственном прообразе $\left(E_{j}^{\sharp}\right)^{i-1}$ исключительного дивизора $E_{j}^{\sharp}$ на $V_{i-1}^{\sharp}$. Пусть целое число $p_{i j}$ при $i>j$ обозначает количество путей

$$
i_{0}=i \rightarrow i_{1} \rightarrow \cdots \rightarrow i_{k}=j, \quad k=1,2, \ldots
$$

из $i$ в $j$ в графе $\Gamma$ нормирования $\nu$. Положим также $p_{i i}=1$. Таким образом,

$$
p_{i j}=\nu_{E_{i}^{\sharp}}\left(E_{j}^{\sharp}\right) .
$$

В силу стандартных формул теории пересечений [16, приложение В] (см. также [18]) получаем

$$
\text { mult }_{\Delta} Z^{*} \geqslant \sum_{i=1}^{N} \mu_{i}^{2} \text {. }
$$


ЛЕмма 9. Выполнена следующая оченка:

$$
\sum_{i=1}^{N} p_{i} \mu_{i}>n\left(a(E) p_{1}+\sum_{i=1}^{N} p_{i}\right)
$$

где $p_{i}=p_{N, i}$ и а(E) обозначает дискрепантность $E$.

ДокАЗАТЕЛЬство. В силу неравенства Нётера-Фано

$$
\nu(\Sigma)>n \text { discrepancy }(\nu) .
$$

Здесь $\nu(\Sigma)=\nu\left(\Sigma^{*}\right)$, поскольку $\Sigma^{*}$ - полный прообраз линейной системы $\Sigma$ на $V^{*}$. С помощью стандартной техники (см. [15], [16], [18]) получаем, что $\nu\left(\Sigma^{*}\right)$ есть левая часть неравенства (27). Вычислим дискрепантность нормирования $\nu$ относительно $V$. Имеем

$$
K_{V^{*}}=f^{*} K_{\widetilde{V}}+\sum_{i \in I_{X}} a\left(E_{i}\right) E_{i}^{*},
$$

так что

$$
a(\nu, V) \geqslant a\left(E, \mathbb{P}^{M-2}\right) \nu\left(E^{*}\right)+a\left(\nu, V^{*}\right) .
$$

Поскольку centre $\left(\nu, V^{*}\right)=\Delta \subset E^{*}$, получаем неравенство

$$
\nu\left(E^{*}\right) \geqslant \nu\left(E_{1}^{\sharp}\right)=p_{1}
$$

(действительно, можно сказать более точно, что

$$
\nu\left(E^{*}\right)=\sum_{B_{i-1} \subset\left(E^{*}\right)^{i-1}} p_{i},
$$

где $\left(E^{*}\right)^{i-1}$ - собственньй прообраз дивизора $E^{*}$ на $V_{i-1}^{\sharp}$, но нам это не нужно). Наконец, все центры $B_{i-1}$ имеют коразмерность два, так что

$$
a\left(\nu, V^{*}\right)=\sum_{i=1}^{N} p_{i},
$$

что и требовалось доказать. Лемма доказана.

СледствиЕ 5. Имеет место следующая оценка:

$$
\nu_{E}(F)>n^{2} \frac{\left(a(E) p_{1}+\sum_{i=1}^{N} p_{i}\right)^{2}}{\sum_{i=1}^{N} p_{i}^{2}} .
$$

ДокАЗАТЕЛЬСТво. Вычислим минимум квадратичной формы $\sum_{i=1}^{N} \mu_{i}^{2}$ на гиперплоскости

$$
\sum_{i=1}^{N} p_{i} \mu_{i}=C .
$$

Минимум достигается при $\mu_{i}=p_{i} \lambda$, где обшая константа $\lambda$ находится из (29). Элементарные вычисления завершают доказательство. 
СлеДСтвИЕ 6. Имеет место следующая оценка:

$$
\nu_{E}(F)>2 n^{2}(a(E)+1) .
$$

ДокАЗАТЕЛЬСТво. Предположим сначала, что $N \geqslant 2$. Раскрывая скобки в формуле (28), получим оценку

$$
\left(a(E) p_{1}+\sum_{i=1}^{N} p_{i}\right)^{2}>2 a(E) p_{1}\left(\sum_{i=1}^{N} p_{i}\right)+\left(\sum_{i=1}^{N} p_{i}\right)^{2} .
$$

Но $p_{1} \geqslant p_{i}$ для всех $i=1, \ldots, N$, так что $p_{1} p_{i} \geqslant p_{i}^{2}$ и первое слагаемое в правой части не меньше, чем

$$
2 a(E) \sum_{i=1}^{N} p_{i}^{2}
$$

По определению чисел $p_{i}$ имеем равенство

$$
p_{1}=\sum_{i \rightarrow 1} p_{i}
$$

так что

$$
\left(\sum_{i=1}^{N} p_{i}\right)^{2} \geqslant p_{1} \sum_{i=1}^{N} p_{i}+\left(\sum_{i \rightarrow 1} p_{i}\right) \sum_{i=1}^{N} p_{i}=2 p_{1} \sum_{i=1}^{N} p_{i} \geqslant 2 \sum_{i=1}^{N} p_{i}^{2}
$$

откуда в силу (28) получаем оценку (30) при $N \geqslant 2$.

Если $N=1$, то $p_{1}=1$ и

$$
\nu_{E}(F)>n^{2}(a(E)+1)^{2}
$$

но $(a(E)+1)^{2}=2 a(E)+a(E)^{2}+1 \geqslant 2 a(E)+2$, что и требуется доказать.

Остается заметить, что оценка (30) в точности означает, что пара $\left(\mathbb{P}^{M-2}, \frac{1}{2 n^{2}} F\right)$ не логканонична в точке $y$.

Доказательство предложения 4 и, тем самым, основной теоремы завершено.

Автор благодарит за финансовую поддержку своих исследований Фонд Гумбольдта и Фонд содействия отечественной науке.

\section{Список литературы}

1. Исковских В.А., Манин Ю.И. Трехмерные квартики и контрпримеры к проблеме Люрота // Матем. сб. 1971. Т. 15. № 1. С. 141-166.

2. Corti A. Singularities of linear systems and 3-fold birational geometry // Explicit Birational Geometry of Threefolds. London Mathematical Society Lecture Notes Series. V. 281. Cambridge University Press, 2000. P. 259-312.

3. Corti A., Mella M. Birational geometry of terminal quartic 3-folds. I. Preprint, arXiv: math. AG/0102096.

4. Corti A., Pukhlikov A., Reid M. Fano 3-fold hypersurfaces // Explicit Birational Geometry of Threefolds. London Mathematical Society Lecture Notes Series. V. 281. Cambridge University Press, 2000. P. 175-258. 
5. Esnault H., Viehweg E. Lectures on vanishing theorems // DMV-Seminar. V. 20. Birkhäuser, 1992.

6. Фултон У. Теория пересечений. М.: Мир, 1989.

7. Gizatullin M. Fano's inequality is a mistake. Preprint, arXiv: math. AG/0202069.

8. Gizatullin M. Fano's inequality is also false for three-dimensional quadric. Preprint, arXiv: math. AG/0202117.

9. Gizatullin M. Fano's inequality is false for a simple Cremona transformation of five-dimensional projective space. Preprint, arXiv: math. AG/0202138.

10. Исковских В. А. Бирациональная жесткость гиперповерхностей Фано в рамках теории Мори // УМН. 2001. Т. 56. №2. С. 3-86.

11. Iskovskikh V.A., Pukhlikov A. V. Birational automorphisms of multi-dimensional algebraic varieties // J. Math. Sci. 1996. V. 82. № 4. P. 3528-3613.

12. Kollár J., et al. Flips and Abundance for Algebraic Threefolds // Asterisque. 1993. V. 211.

13. Kawamata Y. A generalization of Kodaira-Ramanujam's vanishing theorem // Math. Ann. 1982. V. 261. P. 43-46.

14. Pukhlikov A. V. Birational automorphisms of four-dimensional quintics // Invent. Math. 1987. V. 87. P. 303-329.

15. Пухликов А.В. Замечание о теореме В.А. Исковских и Ю.И. Манина о трехмерной квартике // Тр. Матем. ин-та им. В.А. Стеклова РАН. 1995. Т. 208. С. 278-289.

16. Pukhlikov A. V. Birational automorphisms of Fano hypersurfaces // Invent. Math. 1998. V. 134. № 2. P. 401-426.

17. Пухликов А.В. Бирациональные автоморфизмы трехмерных алгебраических многообразий с пучком поверхностей дель Пеццо // Изв. РАН. Сер. матем. 1998. Т. 62. № 1. C. $123-164$.

18. Pukhlikov A. V. Essentials of the method of maximal singularities // Explicit Birational Geometry of Threefolds. London Mathematical Society Lecture Notes Series. V. 281. Cambridge University Press, 2000. P. 73-100.

19. Пухликов А. В. Бирационально жесткие расслоения Фано // Изв. РАН. Сер. матем. 2000. T. 64. № 3. C. 131-150.

20. Pukhlikov A. V. Birationally rigid Fano complete intersections // Crelle J. für die reine und angew. Math. 2001. V. 541. P. 55-79.

21. Пухликов А. В. Бирационально жесткие гиперповерхности Фано с изолированными особенностями // Матем. сб. 2002. Т. 193. №3. С. 135-160.

22. Park J. Birational maps of del Pezzo fibrations // Crelle J. für die reine und angew. Math. 2001. V. 538. P. 213-221.

23. Саркисов В.Г. О структурах расслоений на коники // Изв. АН СССР. Сер. матем. 1982. T. 46. № 2. С. 355-390.

24. Чельцов И. А. О гладкой четырехмерной квинтике // Матем. сб. 2000. Т. 191. № 9 . C. $139-162$.

25. Cheltsov I. A. On the sextic, septic and octic. Preprint, 2000.

26. Чельцов И.А. Логканонические пороги на гиперповерхностях // Матем. сб. 2001. T. 192. № 8 . C. $155-172$.

27. Cheltsov I. A., Park J. Log-canonical thresholds and generalized Eckard points. Preprint, arXiv: math. AG/0003121.

28. Шокуров В. В. Трехмерные логперестройки // Изв. РАН. Сер. матем. 1992. Т. 56. № 1. C. 105-203.

29. Viehweg E. Vanishing theorems // Crelle J. für die reine und angew. Math. 1982. V. 335. P. 1-8.

Математический институт им. В.А. Стеклова РАН

E-mail: pukh@mu.ras.ru

Поступило в редакцию 04.IV.2002 